CLINICAL STUDY

\title{
Analysis of mRNA expression for steroidogenic enzymes in the remaining adrenal cortices attached to adrenocortical adenomas
}

\author{
Kazuto Shigematsu ${ }^{1}$, Takehiro Nakagaki ${ }^{2}$, Naohiro Yamaguchi ${ }^{2}$, Kioko Kawai $^{3}$, Hideki Sakai ${ }^{4}$ \\ and Osamu Takahara ${ }^{5}$ \\ Divisions of ${ }^{1}$ Pathology and ${ }^{2}$ Cellular Molecular and Biology, Nagasaki University Graduate School of Biomedical Sciences, 1-12-4 Sakamoto, Nagasaki \\ 852-8523, Japan, ${ }^{3}$ Nagasaki Prefecture Medical Health Operation Group, Department of Pathology, Isahaya 859-0401, Japan, ${ }^{4}$ Division of Nephro- \\ Urology, Nagasaki University Graduate School of Biomedical Sciences, Nagasaki 852-8523, Japan and ${ }^{5}$ Department of Pathology, Japanese Red-Cross \\ Nagasaki Atomic Bomb Hospital, Nagasaki 852-8511, Japan
}

(Correspondence should be addressed to K Shigematsu; Email: shigek@net.nagasaki-u.ac.jp)

\begin{abstract}
Design and methods: We have recently demonstrated that the adrenal cortices attached to aldosteroneproducing adenoma (APA) contained microscopic subcapsular micronodules suggestive of active aldosterone production. In this study, we used in situ hybridization to investigate the mRNA expression of steroidogenic enzymes in the adrenal cortices attached to cortisol-producing adenoma (CPA) and clinically silent adenoma (non-functioning adenoma; NFA), in addition to APA.

Results: Microscopic subcapsular micronodules, which were several hundreds of micrometers in size and spheroid in shape, were observed in the cortices attached to CPA and NFA, as well as APA, at high frequency. Most of the cortical nodules in zona fasciculata to zona reticularis showed a suppressed steroidogenesis in the cortices attached to adenoma, but some expressed intensely all necessary steroidogenic enzyme mRNAs for cortisol synthesis.

Conclusions: It is thus necessary to keep in mind, on the occasion of subtotal adrenalectomy, that lesions with the potential to later develop into functional adrenocortical nodules may be present in other parts of the ipsilateral or contralateral adrenal cortices.
\end{abstract}

European Journal of Endocrinology 158 867-878

\section{Introduction}

Hyperfunction of the adrenal cortex is associated with either hyperplasia, adenoma, or carcinoma. There are principally three forms of adrenocortical hyperfunction, namely primary aldosteronism (PA), Cushing's syndrome (CS), and congenital adrenal hyperplasia. PA and CS are thought to occur as quite distinct entities. Recently, case reports of adrenocortical adenoma overproducing both aldosterone and cortisol are becoming more frequent $(1,2)$, probably as the result of improvements in examination accuracy. Multiple or bilateral adrenocortical adenomas causing both PA and CS at the same time or at different periods have also been reported (3-5). These findings suggest that the same or a similar pathological condition may exist in the pathogenic background of adrenocortical adenomas, regardless of the type of hormone produced in excess.

In aldosterone-producing adenoma (APA), the remaining adrenal cortices often show hyperplasia of the zona glomerulosa (ZG), and one or more micro- or macronodules. Unlike the cortices adhering to APA, the remaining cortices in cortisol-producing adenoma
(CPA) invariably appear atrophic, but cortical nodules with a diffuse brown or yellow color can also be seen occasionally, especially in cases of adrenocorticotrophin (ACTH)-independent bilateral or multiple adrenocortical adenomas $(6,7)$. Most of the cortical nodules in the cortices attached to functional adrenocortical adenomas have been thought to be incidental and nonfunctional, probably related to vascular sclerosis (8). However, histochemical and electron microscopic studies have shown that some ischemia-related cortical nodules had steroidogenic activity $(9,10)$. We have recently demonstrated that the cortices attached to APA contained not only microscopic subcapsular micronodules suggesting active aldosterone production, as observed in bilateral adrenal hyperplasia (BAH) and unilateral adrenal hyperplasia (UAH), but also cortical nodules suggesting the capability for producing cortisol (11). Hence, the possibility is suggested that buds with autonomous production of aldosterone or cortisol may exist in the cortices attached to APA.

Laparoscopic subtotal adrenalectomy with preservation of functional adrenal tissue has been attempted $(12,13)$. This approach would seem to be successful, 
although the postoperative long-term follow-up is often inadequate $(5,7,14)$. Meanwhile, the recurrence of PA or CS following total or subtotal adrenalectomy has also been reported $(3,15-17)$. Therefore, knowledge of what lesions are present in the remaining cortices is essential. In this study, we used in situ hybridization to examine the expression of mRNAs for steroidogenic enzymes in the remaining adrenal cortices attached to adrenocortical adenomas associated with PA and CS, as well as in clinically silent cases (NF).

\section{Materials and methods}

\section{Tissue samples}

Of the paraffin-embedded human adrenal tissues listed in the Division of Pathology, Nagasaki University Graduate School of Biomedical Sciences, and Red-Cross Nagasaki Atomic Bomb Hospital between 1988 and 2006, 19 and
11 cases of $\mathrm{CP}$ and $\mathrm{NF}$ respectively, were identified (Table 1). Seven cases of PA operated between 2005 and 2006 were also selected (Table 1). As controls, we used five adrenal glands obtained from patients undergoing adrenalectomy together with pancreatectomy or nephrectomy for pancreatic or renal cancer, who did not reveal any endocrine abnormalities. PA was diagnosed on the basis of an elevated plasma aldosterone concentration and suppressed plasma renin activity or concentration. The diagnosis of CS was based on symptoms such as obesity, moon face, buffalo hump, abdominal striae, etc., elevated plasma cortisol concentration with lack of diurnal rhythm and suppressed plasma ACTH level. The exact location of the lesions in all cases of PA and CS was determined by adrenal venous sampling. Clinically silent adenoma non-functioning adenoma (NFA), showing normal range plasma aldosterone and cortisol concentrations, was incidentally detected by computer tomography (CT) scan. However,

Table 1 Clinical data.

\begin{tabular}{|c|c|c|c|c|c|c|c|c|c|c|c|c|}
\hline No. & Age & Clin & BP & Ald & $\mathbf{F}$ & Renin & 17 & АСТН & CR & Dex & Sca & Drug \\
\hline 1 & 37 & PA & $144 / 90$ & 205 & 10.8 & 0.2 & $\mathrm{~N}$ & 18.7 & $\mathrm{~N}$ & $\mathrm{~N}$ & + & $A$ \\
\hline 2 & 38 & PA & 200 & 232 & $N$ & 4 & 0.4 & $\mathrm{~N}$ & $\mathrm{~N}$ & $\mathrm{~N}$ & + & $A$ \\
\hline 3 & 42 & PA & $148 / 110$ & 352 & 13.4 & $<2.3$ & 7.1 & $\mathrm{~N}$ & $\mathrm{~N}$ & $\mathrm{~N}$ & + & A \\
\hline 4 & 48 & PA & $150 / 100$ & 520 & 10.84 & $<2.0$ & 0.3 & 32 & $\mathrm{~N}$ & $\mathrm{~N}$ & + & A \\
\hline 5 & 54 & PA & $160 / 100$ & 500 & $\mathrm{~N}$ & $<0.2$ & $\mathrm{~N}$ & $\mathrm{~N}$ & $\mathrm{~N}$ & $\mathrm{~N}$ & + & A \\
\hline 6 & 51 & PA & $140 / 90$ & 249 & 6.06 & $N$ & $\mathrm{~N}$ & $\mathrm{~N}$ & $\mathrm{~N}$ & $\mathrm{~N}$ & + & A \\
\hline 7 & 52 & PA & $200 / 110$ & 168 & 8.18 & 3.3 & 0.3 & $\mathrm{~N}$ & + & $\mathrm{N}$ & + & $A$ \\
\hline 8 & 27 & CS & $160 / 100$ & $\mathrm{~N}$ & 22.3 & $\mathrm{~N}$ & $\mathrm{~N}$ & ND & - & - & + & B \\
\hline 9 & 51 & CS & $155 / 93$ & $\mathrm{~N}$ & 22.5 & $\mathrm{~N}$ & 9.3 & ND & - & - & + & B \\
\hline 10 & 48 & CS & $135 / 85$ & $\mathrm{~N}$ & 20.2 & $\mathrm{~N}$ & 12.2 & ND & - & - & + & B \\
\hline 11 & 67 & CS & $148 / 96$ & $\mathrm{~N}$ & 19.8 & $\mathrm{~N}$ & 14.3 & ND & - & - & + & B \\
\hline 12 & 47 & CS & $150 / 92$ & $\mathrm{~N}$ & 23.3 & $\mathrm{~N}$ & 11.1 & ND & - & - & + & $A$ \\
\hline 13 & 52 & CS & $160 / 100$ & $\mathrm{~N}$ & 22.7 & $\mathrm{~N}$ & 10.8 & ND & - & - & + & B \\
\hline 14 & 48 & CS & 200 & $\mathrm{~N}$ & 19.3 & $\mathrm{~N}$ & 14.7 & ND & - & - & + & A \\
\hline 15 & 87 & CS & $200 / 100$ & $\mathrm{~N}$ & 19.4 & 2.3 & 7.8 & ND & - & - & + & A \\
\hline 16 & 29 & CS & $105 / 82$ & $<10$ & 22.7 & 3.9 & 15.8 & 3.3 & - & - & + & - \\
\hline 17 & 48 & CS & $147 / 92$ & $N$ & 21.2 & $\mathrm{~N}$ & 9.8 & ND & - & - & + & B \\
\hline 18 & 54 & CS & $158 / 106$ & $\mathrm{~N}$ & 22.5 & $\mathrm{~N}$ & $\mathrm{~N}$ & 5 & - & - & + & - \\
\hline 19 & 37 & CS & 180 & $\mathrm{~N}$ & 25.1 & $\mathrm{~N}$ & 11.6 & ND & - & - & + & B \\
\hline 20 & 45 & CS & $160 / 96$ & 44 & 22.3 & 78.2 & $\mathrm{~N}$ & ND & - & - & + & A \\
\hline 21 & 37 & CS & $155 / 90$ & 32 & 21.5 & 20.6 & 0.7 & ND & - & - & + & B \\
\hline 22 & 32 & CS & $148 / 108$ & $\mathrm{~N}$ & 23.3 & $\mathrm{~N}$ & 15.7 & ND & - & - & + & - \\
\hline 23 & 37 & CS & $145 / 110$ & $\mathrm{~N}$ & 24.2 & $\mathrm{~N}$ & 15 & ND & - & - & + & B \\
\hline 24 & 65 & CS & $200 / 100$ & 30 & 15 & 2.4 & 13.7 & ND & $\mathrm{N}$ & - & + & $A$ \\
\hline 25 & 42 & CS & 150 & *189 & 13.9 & $\mathrm{~N}$ & $\mathrm{~N}$ & ND & - & - & + & - \\
\hline 26 & 38 & CS & $127 / 76$ & $\mathrm{~N}$ & 17.7 & $\mathrm{~N}$ & $\mathrm{~N}$ & ND & - & - & + & - \\
\hline 27 & 67 & NF & $194 / 98$ & $\mathrm{~N}$ & 7.2 & $\mathrm{~N}$ & $\mathrm{~N}$ & $\mathrm{~N}$ & $\mathrm{~N}$ & $\mathrm{~N}$ & $\mathrm{~N}$ & $\mathrm{~N}$ \\
\hline 28 & 48 & NF & $128 / 88$ & 10 & 14.1 & 0.2 & $\mathrm{~N}$ & 14 & + & $\mathrm{N}$ & $\mathrm{N}$ & - \\
\hline 29 & 74 & NF & $136 / 99$ & 43 & 11.1 & 3.5 & $\mathrm{~N}$ & $\mathrm{~N}$ & + & $\mathrm{N}$ & $\mathrm{N}$ & - \\
\hline 30 & 58 & NF & $127 / 76$ & $\mathrm{~N}$ & 17.7 & $\mathrm{~N}$ & $\mathrm{~N}$ & ND & - & $\mathrm{N}$ & $\mathrm{N}$ & - \\
\hline 31 & 65 & NF & $125 / 87$ & 2.2 & 5.9 & 6.5 & $\mathrm{~N}$ & $\mathrm{~N}$ & $\mathrm{~N}$ & $\mathrm{~N}$ & $\mathrm{~N}$ & - \\
\hline 32 & 54 & NF & $195 / 101$ & 10 & 17.8 & $\mathrm{~N}$ & $\mathrm{~N}$ & $<5.0$ & $\mathrm{~N}$ & $\mathrm{~N}$ & + & A \\
\hline 33 & 66 & NF & $160 / 90$ & 120 & 6.6 & $\mathrm{~N}$ & $\mathrm{~N}$ & 20 & + & $\mathrm{N}$ & $\mathrm{N}$ & $B$ \\
\hline 34 & 40 & NF & $144 / 99$ & 40 & 15.7 & $\mathrm{~N}$ & $\mathrm{~N}$ & $<5.0$ & $\mathrm{~N}$ & $\mathrm{~N}$ & + & B \\
\hline 35 & 31 & NF & $110 / 80$ & $\mathrm{~N}$ & 6.5 & $\mathrm{~N}$ & $\mathrm{~N}$ & $\mathrm{~N}$ & $\mathrm{~N}$ & $\mathrm{~N}$ & $\mathrm{~N}$ & - \\
\hline 36 & 56 & NF & $204 / 100$ & 59 & 14.3 & $\mathrm{~N}$ & $\mathrm{~N}$ & 11 & - & $\mathrm{N}$ & + & A \\
\hline 37 & 28 & NF & $115 / 66$ & 53 & 18.2 & 0.9 & 6.2 & ND & + & $\mathrm{N}$ & + & - \\
\hline
\end{tabular}

Clin, clinical diagnosis; PA, primary aldosteronism; CS, Cushing's syndrome; NF, clinical silent; BP, blood pressure (mmHg); Ald, plasma aldosterone (29.9-159 pg/ml, *35.7-240 pg/ml), F, plasma cortisol (6.2-19.4 $\mathrm{gg} / \mathrm{ml}) ;$ renin, plasma renin (2.5-21.4 pg/ml); 17, 17-OHCS (2.2-7.3 mg/day); ACTH, plasma ACTH $(7-56 \mathrm{pg} / \mathrm{ml}), \mathrm{CR}$, circadian rhythm: Dex, dexamethasone suppression test; Sca, isotope accumulation on an ${ }^{131}$-aldosterol scintigram; Drug, anti-hypertensive agents, A (spironolactone), B (calcium antagonists); ND, not detected; N, not determined. 
cases 30, 32, 34, 36, and 37 showed low levels of plasma ACTH, loss of circadian rhythm, or isotope accumulation on an $I^{131}$-adosterol scintigram (Table 1). As shown in Table 2, all adrenal tumors examined were microscopically confirmed to be adrenocortical adenomas. All patients signed a form of informed consent prepared in accordance with the rules outlined by the Nagasaki University Ethics Committee.

\section{In situ hybridization}

cDNA fragments of steroidogenic acute regulatory protein (StAR), CYP11A1, HSD3B2, CYP11B, CYP17A1, CYP21A2, sulfotransferase (SULT2A1), and melanocortin 2 receptor (MC2R), which were encoding human StAR protein, cholesterol side-chain cleavage enzyme, $3 \beta$ hydroxysteroid dehydrogenase, $11 \beta$-hydroxylase and/or aldosterone synthase, 17 $\alpha$-hydroxylase, 21-hydroxylase, DHEA sulfotransferase, and receptor for ACTH, respectively, were obtained by reverse transcriptase-PCR and subcloned into pT-NOT vector $(11,18)$. Antisensestrand cRNAs were synthesized using Digoxigenin (DIG)UTP (Roche Diagnostics) with $\mathrm{T}_{3}$ or $\mathrm{T}_{7}$ RNA polymerase (Takara, Otsu, Japan). The probe CYP11B used in this study could not discriminate between 11ß-hydroxylase and aldosterone synthase, since the nucleotide sequences of CYP11B1 and CYP11B2 encoding 11ß-hydroxylase and aldosterone synthase are $95 \%$ identical $(11,19)$.

In situ hybridization was performed as described previously $(11,18)$. Briefly, $3 \mu \mathrm{m}$ thick sections were hybridized with DIG-labeled cRNA probes at $42{ }^{\circ} \mathrm{C}$ for $16 \mathrm{~h}$ and finally washed in $0.2 \times \mathrm{SCC}$ at $50^{\circ} \mathrm{C}$ for $20 \mathrm{~min}$. Hybridization signals were immunologically detected with alkaline phosphatase-conjugated antiDIG Fab fragments (diluted 1:500; Roche Diagnostics). The specificity of the signals was examined using three negative control procedures; a) hybridization with sense cRNA probe, b) pretreatment of tissue sections with

Table 2 Expression pattern in the adrenal cortices attached to APA, CAP, and NFA.

\begin{tabular}{|c|c|c|c|c|c|c|c|c|c|}
\hline No. & Diag & Site & Size & Atrophy & (a) & (b) & (c) & (d) & (e) \\
\hline 1 & APA & $\mathrm{R}$ & 2 & - & + & + & + & + & - \\
\hline 2 & APA & L & 1.5 & - & + & + & + & + & - \\
\hline 3 & APA & $\mathrm{R}$ & 1.5 & - & + & + & + & + & - \\
\hline 4 & APA & L & 0.9 & - & + & + & + & + & + \\
\hline 5 & APA & $\mathrm{R}$ & 2.2 & - & + & + & + & + & + \\
\hline 6 & APA & L & 1.2 & - & - & + & + & + & + \\
\hline 7 & APA & $R$ & 2 & + & + & + & + & + & + \\
\hline 8 & CPA & L & 3 & + & + & + & - & - & + \\
\hline 9 & CPA & L & 3 & + & + & + & - & - & + \\
\hline 10 & CPA & $\bar{L}$ & 3.4 & + & - & - & - & - & + \\
\hline 11 & CPA & L & 3.5 & + & + & - & - & - & + \\
\hline 12 & CPA & L & 4.2 & + & - & + & - & - & + \\
\hline 13 & CPA & L & 4.1 & + & + & + & + & - & + \\
\hline 14 & CPA & $\mathrm{R}$ & 4 & + & + & + & - & - & + \\
\hline 15 & CPA & L & 4 & + & + & + & + & - & + \\
\hline 16 & CPA & L & 4.5 & + & - & - & - & - & - \\
\hline 17 & CPA & $\bar{L}$ & 3 & + & + & + & - & - & + \\
\hline 18 & CPA & L & 3.2 & + & + & + & - & - & - \\
\hline 19 & CPA & L & 3 & + & + & + & + & - & - \\
\hline 20 & CPA & $\bar{L}$ & 2.5 & + & + & + & + & - & + \\
\hline 21 & CPA & L & 3 & + & - & + & + & - & + \\
\hline 22 & CPA & $\mathrm{R}$ & 2.4 & + & + & + & + & + & - \\
\hline 23 & CPA & L & 3.7 & + & + & + & + & - & + \\
\hline 24 & CPA & $\mathrm{R}$ & 3 & + & + & + & + & - & - \\
\hline 25 & CPA & L & 3.3 & + & + & + & + & - & - \\
\hline 26 & CPA & L & 3.8 & + & + & + & + & - & - \\
\hline 27 & NFA & L & 3 & - & + & + & + & + & + \\
\hline 28 & NFA & $\mathrm{R}$ & 4 & + & + & - & - & - & + \\
\hline 29 & NFA & $\mathrm{L}$ & 4 & - & - & + & - & + & + \\
\hline 30 & NFA & $\mathrm{R}$ & 3 & + & + & + & - & - & + \\
\hline 31 & NFA & $\mathrm{L}$ & 4 & - & + & + & - & + & - \\
\hline 32 & NFA & L & 5 & + & - & - & - & - & + \\
\hline 33 & NFA & $\mathrm{R}$ & 3 & + & + & - & - & - & + \\
\hline 34 & NFA & L & 3 & + & + & + & + & - & + \\
\hline 35 & NFA & $\bar{L}$ & 3.2 & - & + & + & - & - & + \\
\hline 36 & NFA & L & 3.2 & + & + & + & - & - & + \\
\hline 37 & NFA & $\mathrm{R}$ & 3.5 & + & - & + & - & - & + \\
\hline
\end{tabular}

a, nodules suggesting the capability for producing aldosterone; $b$, nodules expressing an intense CYP17A1 but not SULT2A1; c, nodules co-expressing SULT2A1; d, nodules co-expressing MC2R; e, nodules with decreased expression for steroidogenic enzymes; Diag, pathological diagnosis; APA, aldosteroneproducing adenoma; CPA, cortisol-producing adenoma; NFA, non-functioning adenoma; 'Atrophy' indicates the pathological finding in the attached remaining adrenal cortex. 
RNase A, and c) displacement by addition of excess unlabeled antisense probe.

\section{Real-time PCR}

To discriminate between the expression of CYP11B1 and CYP11B2 mRNAs, we performed real-time PCR. Frozen adrenal glands attached to adrenocortical adenomas were cut into $7 \mu \mathrm{m}$ thick and consecutively $300 \mu \mathrm{m}$ thick sections in a cryostat. The former were used to examine the expression of HSD3B2 and CYP17A1 mRNAs by in situ hybridization, to determine the presence of subcapsular micronodules, and the latter were stored at $-80^{\circ} \mathrm{C}$ until RNA extraction. Under stereomicroscope, the tissues containing subcapsular micronodules were enucleated with a $400 \mu \mathrm{m}$ needle (inside diameter) from frozen $300 \mu \mathrm{m}$ thick sections of adrenal glands. Total RNA was collected using GenElute Mammalian Total RNA kit (Sigma-Aldrich Inc). Approximately $300 \mathrm{ng}$ of total RNA was extracted from ten enucleated tissues. The RT reaction proceeded at $42{ }^{\circ} \mathrm{C}$ for 60 min with $200 \mathrm{ng}$ total RNA in a reaction mixture containing $50 \mathrm{ng}$ random primer (Life Technologies), $0.5 \mathrm{mM}$ deoxy-NTPs, $10 \mathrm{mM}$ dithiothreitol, $1 \times$ reaction buffer, $50 \mathrm{U}$ RNase inhibitor (Takara), and $100 \mathrm{U}$ RT enzyme (Life Technologies) in a final volume of $20 \mu \mathrm{l}$. The samples were heated at $95^{\circ} \mathrm{C}$ for $10 \mathrm{~min}$ to inactivate RT activity and denature the RNA-cDNA hybrids. LightCycler Quick System 330 (Roche Diagnostic Co.) was used for real-time PCR. Sequence-specific primers were designed, as previously described (20), and assigned the following GenBank accession numbers: CYP11B1 (X55764: 1435-1460 and 1534-1513), CYP11B2 (X54741: 2637-2658 and 2724-2699). Expression levels were standardized by $18 \mathrm{~S}$ rRNA (M10098: 124-148 and 256-232). All products were checked by electrophoresis using 3\% agarose gels and ethidium bromide staining with u.v. visualization to ensure the specificity of the PCR products and the absence of nonspecific bands. Relative quantitation of gene expression was performed using the relative standard curve method. All real-time PCRs were done in triplicate. The sequence of PCR products was analyzed using ABI PRISM 3100 Genetic Analyzer (Applied Biosystems, Tokyo, Japan), in accordance with the manufacturer's recommendations. The results were shown as mean \pm s.E.M.

\section{Results}

\section{Expression of steroidogenic enzyme mRNAs in control adrenal cortices}

Expression levels were heterogeneous within each adrenal cortex and differed among cases, but the site of expression of each mRNA was constant. StAR mRNA was found throughout the whole adrenal cortex, but not in the medulla (Fig. 1A and B). CYP11A1 and CYP17A1 mRNAs were expressed mainly in the zona fasciculata (ZF), followed by the zona reticularis (ZR; Fig. 1C and F). HSD3B2 mRNA was expressed in ZG and ZF, but only
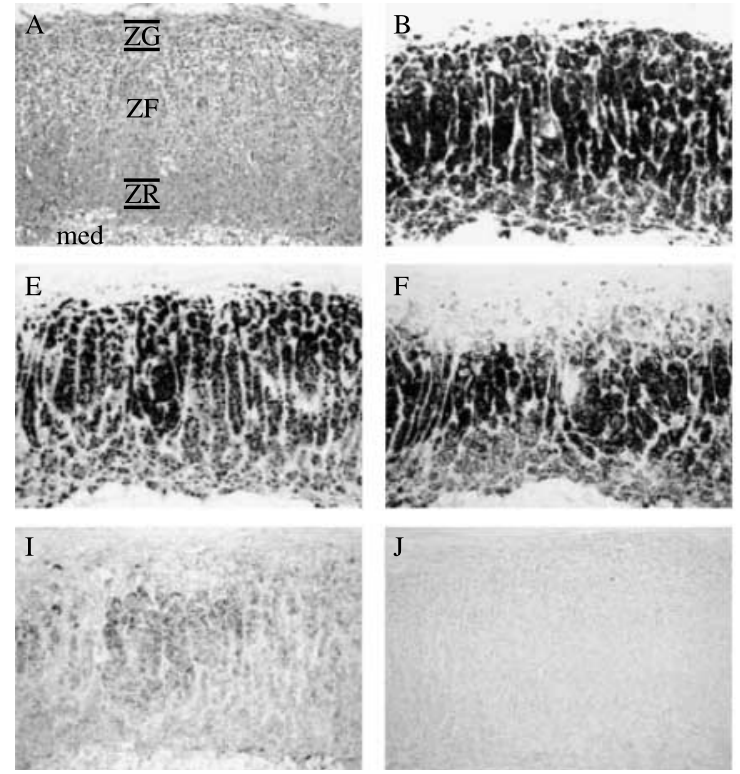
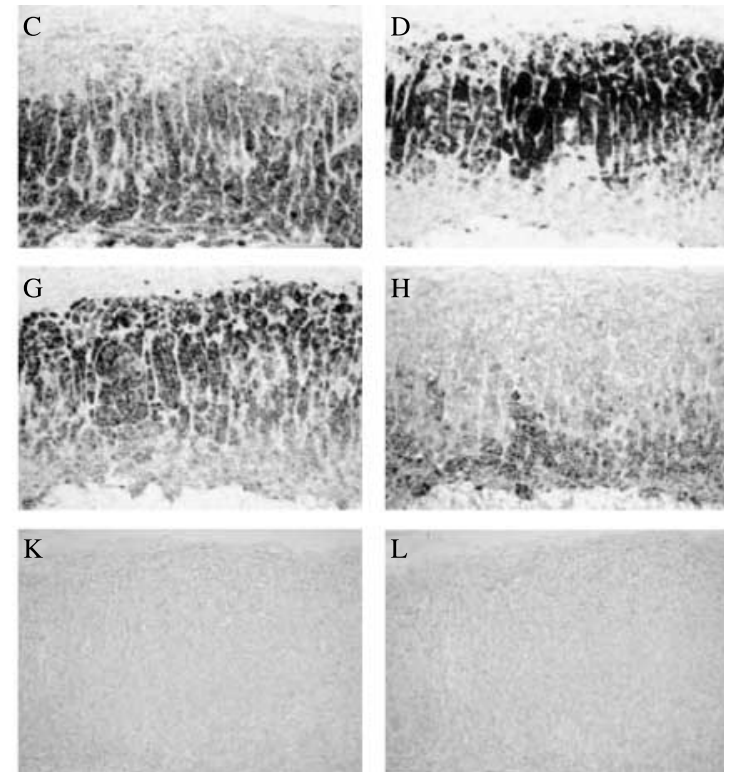

Figure 1 Expression of steroidogenic enzymes in the control. In the controls, StAR (B) mRNA was found throughout adrenal cortex. The expression of CYP11A1 (C) and CYP17A1 (F) mRNAs is found in ZF and ZR. HSD3B2 (D) mRNA is expressed in ZG and ZF, and sporadically expressed in ZR. CYP11B (E) and CYP21A2 (G) mRNAs are intensely expressed in ZG and ZF, followed by ZR. SULT2A1 (H) mRNA expression is present mainly in ZR and ZF. MC2R mRNA is expressed faintly in ZF and ZR (I). (J-L) show the negative controls; hybridization with StAR sense probe $(\mathrm{J})$, pretreatment of tissue sections with RNase A before hybridization with StAR antisense probe $(K)$, and displacement by addition of excess unlabeled StAR antisense probe (L). (A) Stained with hematoxylin-eosin are consecutive sections of (B). Med, medulla. 
sporadically in ZR (Fig. 1D). CYP11B and CYP21A2 mRNAs were intensely expressed in ZG and ZF, followed by ZR (Fig. 1E and G). SULT2A1 mRNA expression was present mainly in ZR and extended to ZF (Fig. 1H). MC2R mRNA was expressed faintly in ZF and ZR (Fig. 1I). In three cases, there were one or two microscopic subcapsular micronodules expressing StAR, CYP11A1, HSD3B2, CYP11B, and CYP21A2, but not CYP17A1 (data not shown). These microscopic subcapsular micronodules were several hundreds of micrometers in size and spheroid in shape. Cortical nodules in ZF-ZR expressing intensely for CYP17A1 mRNA were found in three cases, among which one expressed SULT2A1 mRNA (data not shown). There was no evidence of specific hybridization signals in the negative control (Fig. 1J-L).

\section{Expression of steroidogenic enzyme mRNAs in the adrenal cortices attached to APA}

The remaining cortices were of normal size and there was no suppression of expression for steroidogenic enzyme mRNAs (Fig. 2A-F). SULT2A1 and MC2R mRNAs were expressed in ZF and ZR (Fig. 2G and $\mathrm{H}$ ) as in the control cortices. As expected, six cases $(85.7 \%)$ showed microscopic subcapsular micronodules suggesting aldosterone production, composed of spironolactone body-containing cells (Table 2; Fig. 3A-F). Cortical nodules with expression of StAR, CYP11A1, HSD3B2, CYP17A1, CYP11B, and CYP21A2 mRNAs, suggesting the capability for producing cortisol, were present in all cases (Table 2; Fig. 3G-K). The majority of these nodules also co-expressed SULT2A1 and MC2R mRNAs (Table 2; Fig. 3L).

\section{Expression of steroidogenic enzyme mRNAs in the adrenal cortices attached to CPA}

The remaining cortices were atrophic, and consisted of mainly clear-type cells, in which the expression for steroidogenic enzymes was suppressed in general
(Fig. 4). SULT2A1 mRNA was scattered throughout $\mathrm{ZR}$ and ZF cells. MC2R mRNA expression was also suppressed, but was present in a few cells of ZF and ZR (data not shown). Microscopic subcapsular micronodules (Table 2; Fig. 4) were noted in 15 cases (78.9\%). Most of the cortical nodules in $\mathrm{ZF}-\mathrm{ZR}$ revealed a decreased expression for steroidogenic enzymes. However, 16 out of 19 cases $(84.2 \%)$ contained cortical nodules expressing the mRNAs of steroidogenic enzymes necessary for the production of cortisol (Table 2; Fig. 5A-F). The composition of cells in some nodules showed pleomorphic nuclei and either eosinophilic or fine lipid-rich cytoplasm, as observed in primary pigmented nodular adrenocortical disease. Of the 16 cases, 9 had nodules that co-expressed SULT2A1 mRNA (Fig. 5F) but not MC2R mRNA (Table 2). Only in case 22 did the nodule express both SULT2A1 and MC2R mRNAs (Table 2). Four cases of adrenal cortical hyperplasia with an unencapsulated extension into the periadrenal fat were composed of clear-type cells expressing HSD3B2 but not CYP17A1, and small compact-type cells expressing CYP17A1 but not HSD3B2 (Fig. 5G and H). In three cases, pigmented cortical nodules were found, in which StAR, CYP11A1, CYP17A1, and SULT2A1 mRNAs were expressed, but not HSD3B2, CYP11B, CYP21A2, or MC2R (data not shown).

\section{Expression of steroidogenic enzyme mRNAs in the adrenal cortices attached to NFA}

Atrophic cortices were found in 7 out of 11 cases. The expression of steroidogenic enzyme mRNAs was comparatively conserved. On the other hand, the suppressed expression of SULT2A1 and MC2R mRNAs was evident in the majority of cases. Microscopic subcapsular micronodules were found in $8(72.7 \%)$ out of 11 cases (Table 2). Cortical nodules expressing the mRNAs of steroidogenic enzymes necessary for production of cortisol were noted in eight cases (72.7\%),
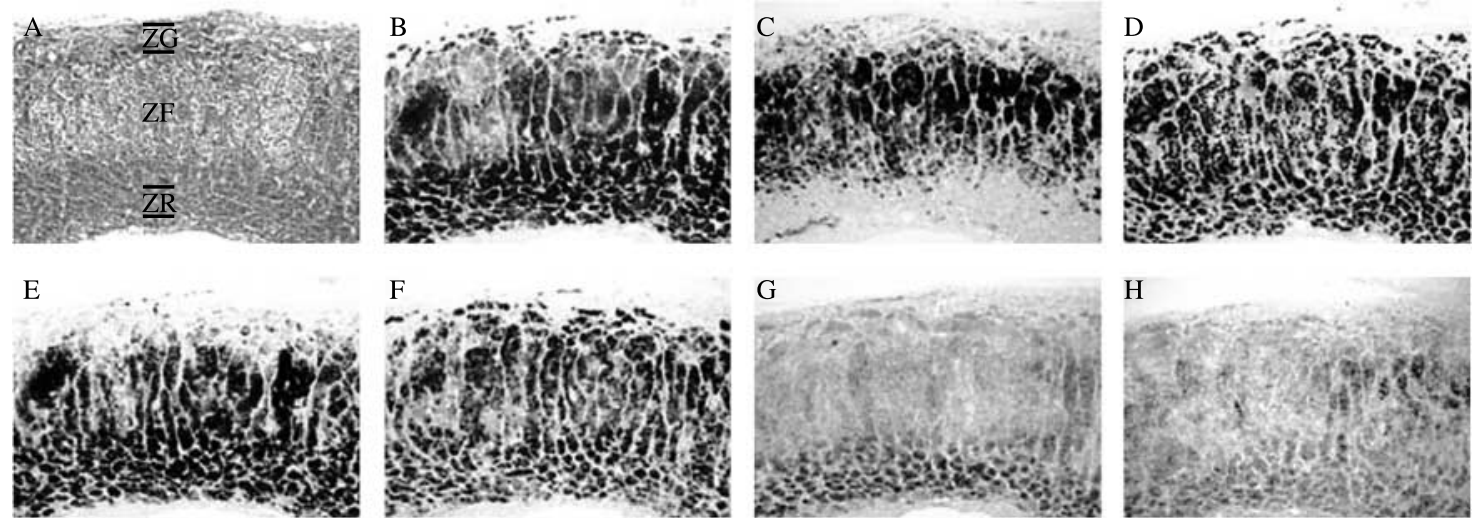

$\mathrm{G}$
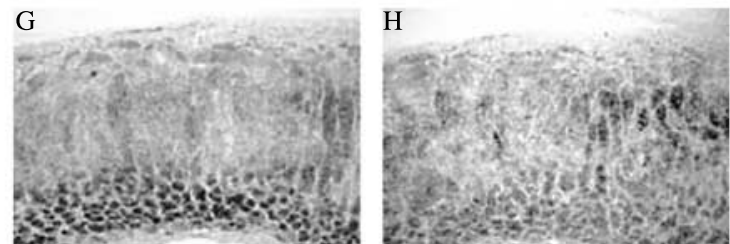

Figure 2 Expression of steroidogenic enzymes in the adrenal cortices attached to APA. The adrenal cortices attached to APA cells express steroidogenic enzymes including HSD3B2 $(C)$, SULT2A1 $(G)$, and MC2R $(H)$ at the same level as the controls. (A) Stained with hematoxylin-eosin are consecutive sections of (B). (B) StAR; (D), CYP11B; (E), CYP17A1; (F), CYP21A2. 

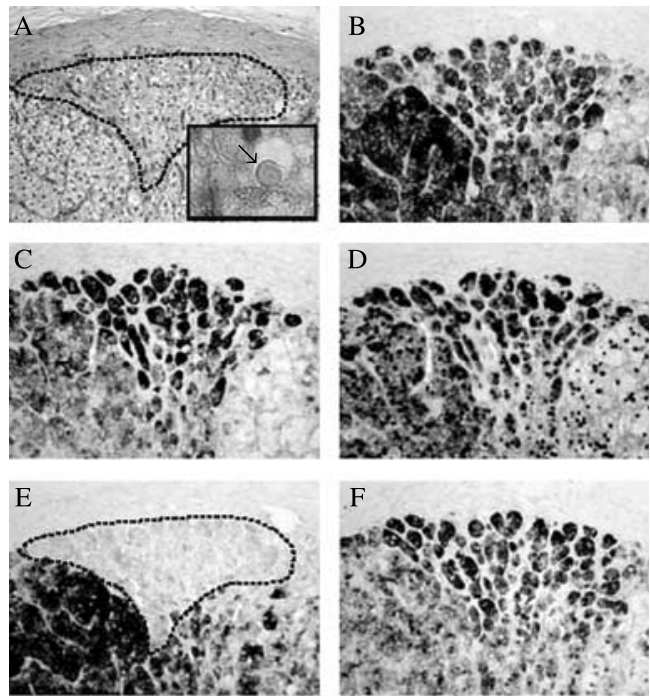

G

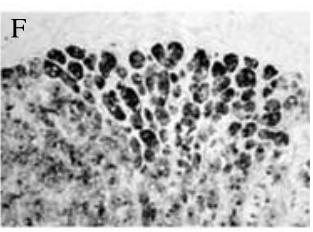

$\mathrm{H}$
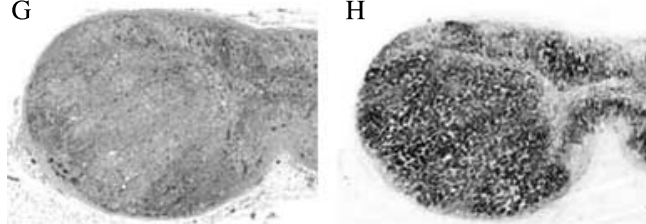

I
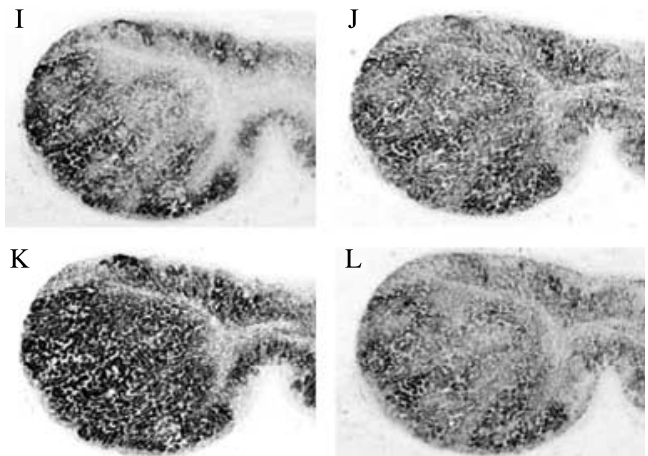

Figure 3 Expression of steroidogenic enzymes in the adrenal cortices attached to APA. Microscopic subcapsular micronodule with an intense expression of StAR (B), HSD3B2 (C), CYP11B (D), and CYP21A2 (F), but not CYP17A1 (E: round dotted lines), can be seen. The majority of subcapsular micronodules in the adrenal cortices attached to APA are composed of spironolactone body-containing cells (A: arrow in square box). The cortical nodule in ZF-ZR expresses StAR (H), CYP11A1, HSD3B2 (I), CYP11B (J), CYP17A1 (K), and CYP21A2 mRNAs, suggesting the capability for cortisol production, and expression of MC2R (L) mRNA. (A) and (G) stained with hematoxylineosin are consecutive sections of $(B)$ and $(H)$ respectively.

but, among these, SULT2A1 or MC2R co-expression was confirmed only in four cases (Table 2).

\section{Real-time PCR}

The tissues containing subcapsular micronodules expressing HSD3B2 but not CYP17A1 were examined (Fig. 6A-C). As controls, APA tissues and the capsular domain tissues without subcapsular micronodules were used. CYP11B1 and CYP11B2 mRNAs were detectable in all tissues examined. Figure 6D shows amplification curves for CYP11B1, CYP11B2, and 18S rRNA. Agarose gels produced by electrophoresis indicated the single bands for CYP11B1, CYP11B2, and 18S rRNA PCR products (Fig. 6E), and the sequencing analysis demonstrated that the PCR products corresponded to CYP11B1 and CYP11B2 genes (data not shown). The ratio of CYP11B1/18S rRNA in the tissues containing subcapsular micronodules $\left(2.44 \times 10^{-3}\right)$ was slightly elevated, when compared with that in APA tissues $(n=4 ; 1.56 \pm$ $0.09 \times 10^{-3}$ ) and the capsular domain tissues without subcapsular micronodules $\left(n=3 ; 1.88 \pm 0.09 \times 10^{-3}\right)$ (Fig. 7). On the other hand, the ratio of CYP11B2/18S rRNA in the tissues containing micronodules $(1.81 \times$ $10^{-4}$ ) was situated between the levels of APA tissues $\left(n=4 ; 3.84 \pm 0.49 \times 10^{-4}\right)$ and the capsular domain tissues without subcapsular micronodules $(n=3$; $\left.0.36 \pm 0.01 \times 10^{-4}\right)$ (Fig. 7).

\section{Discussion}

In this study, we examined the mRNA expression of steroidogenic enzymes in adrenal cortices attached to adrenocortical adenomas associated with PA and CA, as well as clinically silent cases, by in situ hybridization. The results in the control adrenal glands were similar to those of previous reports $(11,21-23)$. Additionally, the distribution of mRNA expression corresponded well with the zonal functions of the adrenal cortex (24), although detection of mRNA expression was not necessarily equivalent to the actual steroidogenic activity of tissues examined (25). The expression signals obtained in this study were specific and not false-positive, as demonstrated by three negative control studies.

The remaining cortices in CPA were atrophic, reflecting the undetectable or very low levels of plasma ACTH resulting from the excessive neoplastic production of cortisol. The circulating ACTH concentrations also correlated well with the expression levels of SULT2A1 and MC2R mRNAs. In the atrophic cortices, the expression of SULT2A1 and MC2R mRNAs was suppressed, as described previously (23), while the cortices attached to APA were not atrophic and exhibited no suppression of either SULT2A1 or MC2R mRNAs. Atrophy was also observed in 7 out of the 11 cases assumed to be NFA. The past few years have seen an increase in the number of reports of adrenocortical adenoma associated with preclinical CS identified in cases previously regarded as NFA $(26,27)$. Cases 30, 32, 34, 36, and 37 showed low levels of plasma ACTH, loss of circadian rhythm or a significant isotope accumulation on an $\mathrm{I}^{131}$-aldosterol scintigram, providing the opportunity to correlate function with anatomic abnormalities (28). Hence, the possibility that at least these cases might actually be preclinical CS could not be ruled out, although the examinations necessary for this diagnosis were not adequate. 

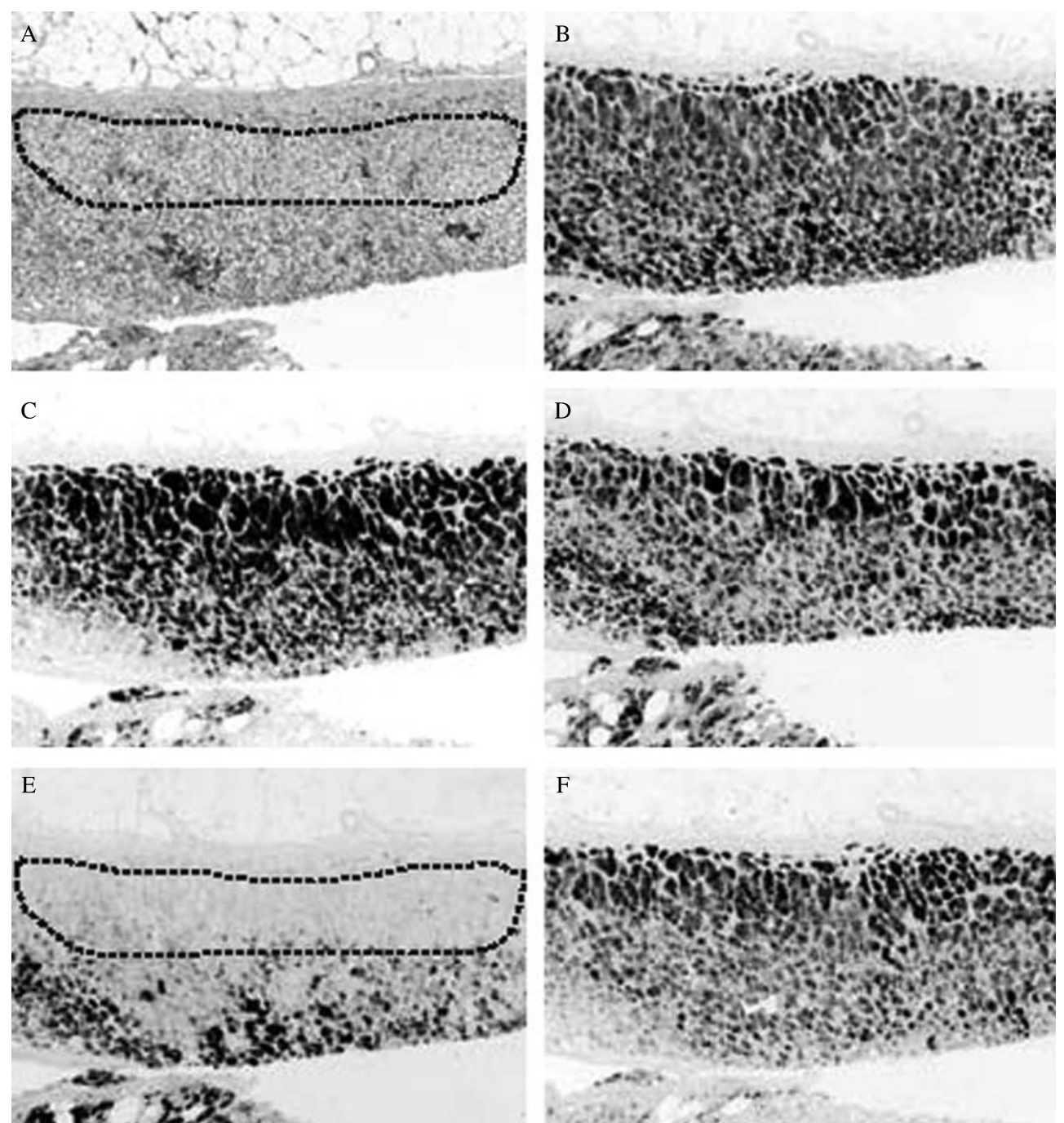

Figure 4 Expression of steroidogenic enzymes in the adrenal cortex attached to CPA. In the adrenal cortices attached to CPA, the expression for steroidogenic enzymes is suppressed, but is comparatively conserved. In microscopic subcapsular micronodule, the expression of StAR (B), HSD3B2 (C), CYP11B (D), and CYP21A2 (F), but not CYP17A1 (E: round dotted lines), can be seen. (A) stained with hematoxylin-eosin are consecutive sections of $(B)$.

We demonstrated that some of the subcapsular micronodules intensely expressed the mRNAs of steroidogenic enzymes necessary for aldosterone production. Regardless of the size of the adenoma, subcapsular micronodules expressing StAR, CYP11A1, HSD3B2, CYP11B, and CYP21A2, but not CYP17A1, were present in the cortices attached to APA at high frequency, as described previously (11). Unexpectedly, similar subcapsular micronodules were equally likely to be observed even in the controls and the cortices attached to CPA and NFA, although there were fewer subcapsular micronodules per case. As these micronodules were observed even in the controls showing no endocrine abnormalities, the possibility existed that the number of aldosterone-producing micronodules might be insufficient to provoke symptoms, or that the micronodules themselves might be unrelated to aldosterone production. However, subcapsular micronodules with the same morphology and expression pattern have been shown to be the main foci of aldosterone synthesis in some UAH cases (11). Additionally, the presence of spironolactone bodies in APA cases is proof that such microscopic subcapsular micronodules had enhanced steroidogenic activity, because spironolactone, a competitive antagonist of aldosterone receptors (29), directly interferes with the overproduction of aldosterone through inhibition of 11ß-hydroxylation and 18-hydroxylation in human adrenal cortical tissue (30). These observations support the possibility that some of the subcapsular micronodules might be an initial focus for the later development into hyperplasia or adenoma, causing PA $(11,31)$. By means of in situ 

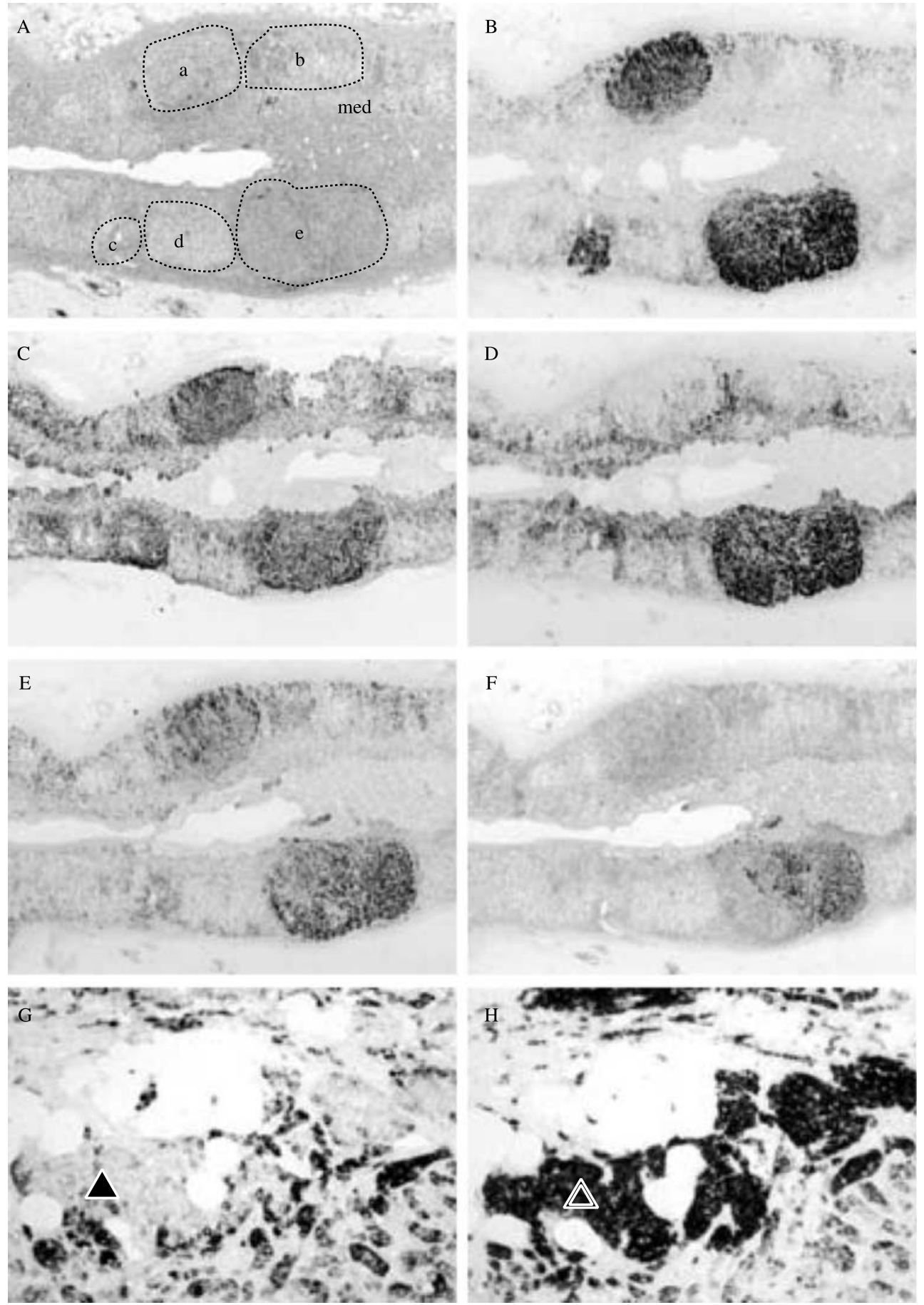

Figure 5 Expression of steroidogenic enzymes in the adrenal cortices attached to CPA. (A-F) indicate five cortical nodules $(a-e)$ in the attached adrenal cortex. (a) and (c) show the expression of HSD3B2 (B), CYP11B (C), and CYP21A2 (E) mRNAs, but absent or sporadic in the expression of CYP17A1 (D) mRNA, suggesting the capability for aldosterone production. (b) and (d) show decreased expression of steroidogenic enzymes. (e) expresses all steroidogenic enzymes including SULT2A1 (F) mRNA, suggesting the capability for cortisol synthesis. The adrenal cortical hyperplasia with unencapsulated extension into the periadrenal fat is composed of cells expressing HSD3B2 $(\mathrm{G})$ but not CYP17A1 $(\mathrm{H})$ and the cells expressing CYP17A1 but not HSD3B2 (G and H: triangles). (A) Stained with hematoxylineosin are consecutive sections of $(B)$. med, medulla. 

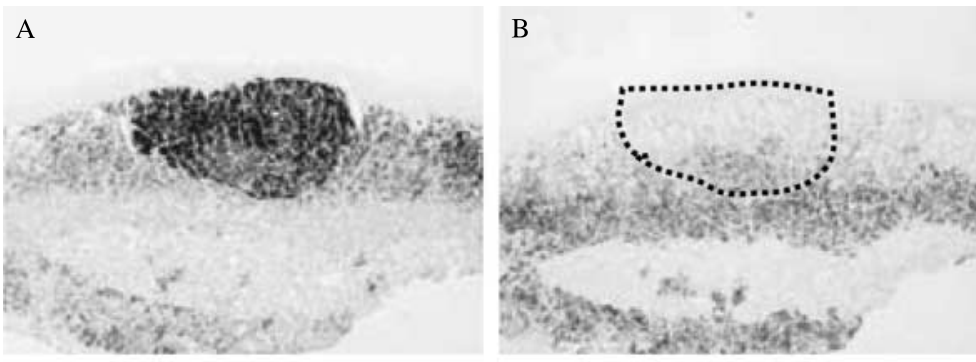

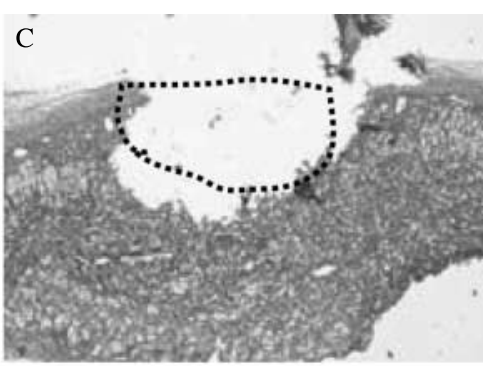

$\mathrm{D}$
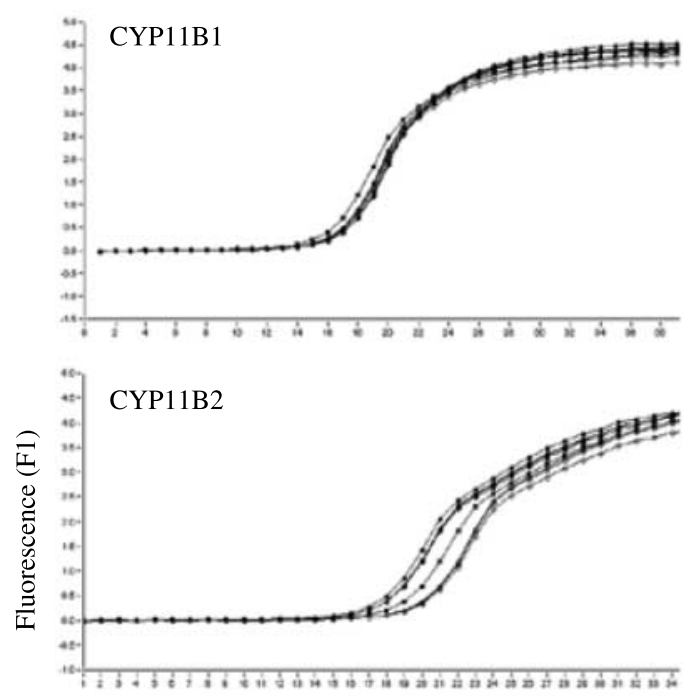

$18 \mathrm{~S}$ rRNA

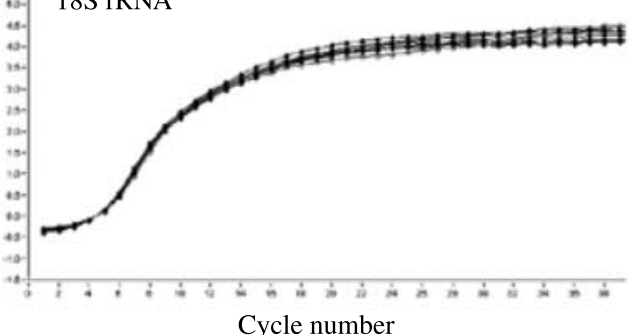

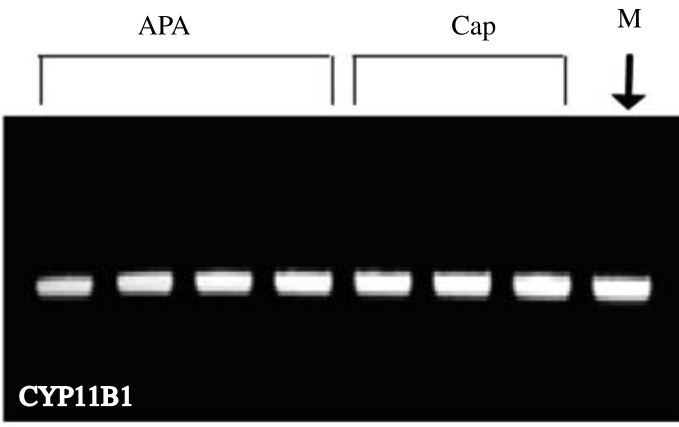
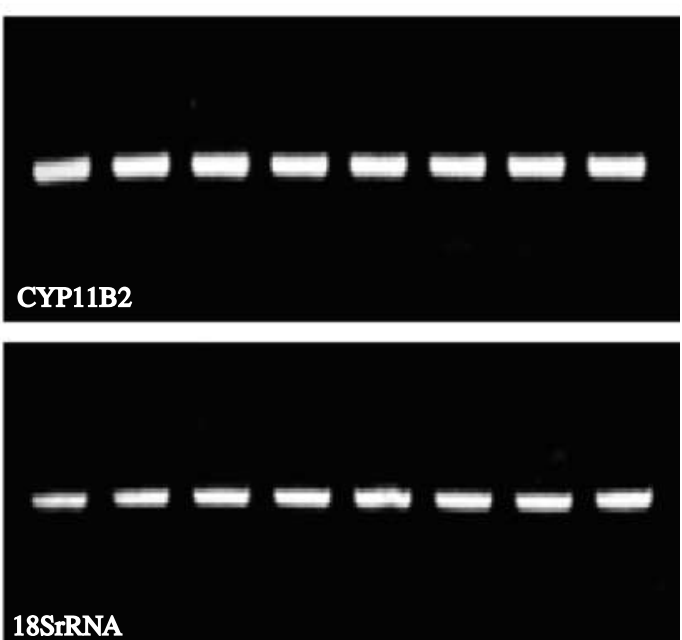

Figure 6 Analysis of CYP11B1 and CYP11B2 mRNA expression by real-time PCR. The tissues containing subcapsular micronodules expressing HSD3B2 (A) but not CYP17A1 (B, round dotted lines) are isolated. $(\mathrm{C})$ is the section stained with toluidine blue after enucleation of the tissue containing subcapsular micronodule. (D) shows amplification curves for CYP11B1, CYP11B2, and 18S rRNA. (E) indicates the ethidium bromide staining of the $3 \%$ agarose gels. Cap, the capsular domain tissues without subcapsular micronodules; M, the tissues containing subcapsular micronodule.

hybridization, we could not confirm that excess aldosterone was actually synthesized in these micronodules, since the probe used for CYP11B could not distinguish $11 \beta$-hydroxylase from aldosterone synthase, being the enzyme for the final step of aldosterone synthesis. Enberg et al. (31) have reported the expression of CYP11B2 mRNA in ZG by in situ hybridization using a radiolabeled oligonucleotide probe, and the autoradiogram exhibited seemed to indicate the presence of subcapsular micronodules expressing CYP11B2 mRNA. Hence, we performed real-time PCR to confirm the expression of
CYP11B2 mRNA in the subcapsular micronodules. The level of CYP11B2 mRNA in the tissues containing micronodules was about 5.5-fold higher than that in the capsular domain tissues without micronodules, although the level of CYP11B2 mRNA was about twofold lower than that in APA tissues. These results indicate that, if not all, at least some subcapsular micronodules themselves expressed levels of CYP11B2 mRNA sufficient for aldosterone production.

In older individuals and in association with hypertension, there is an increased frequency of cortical nodules in 

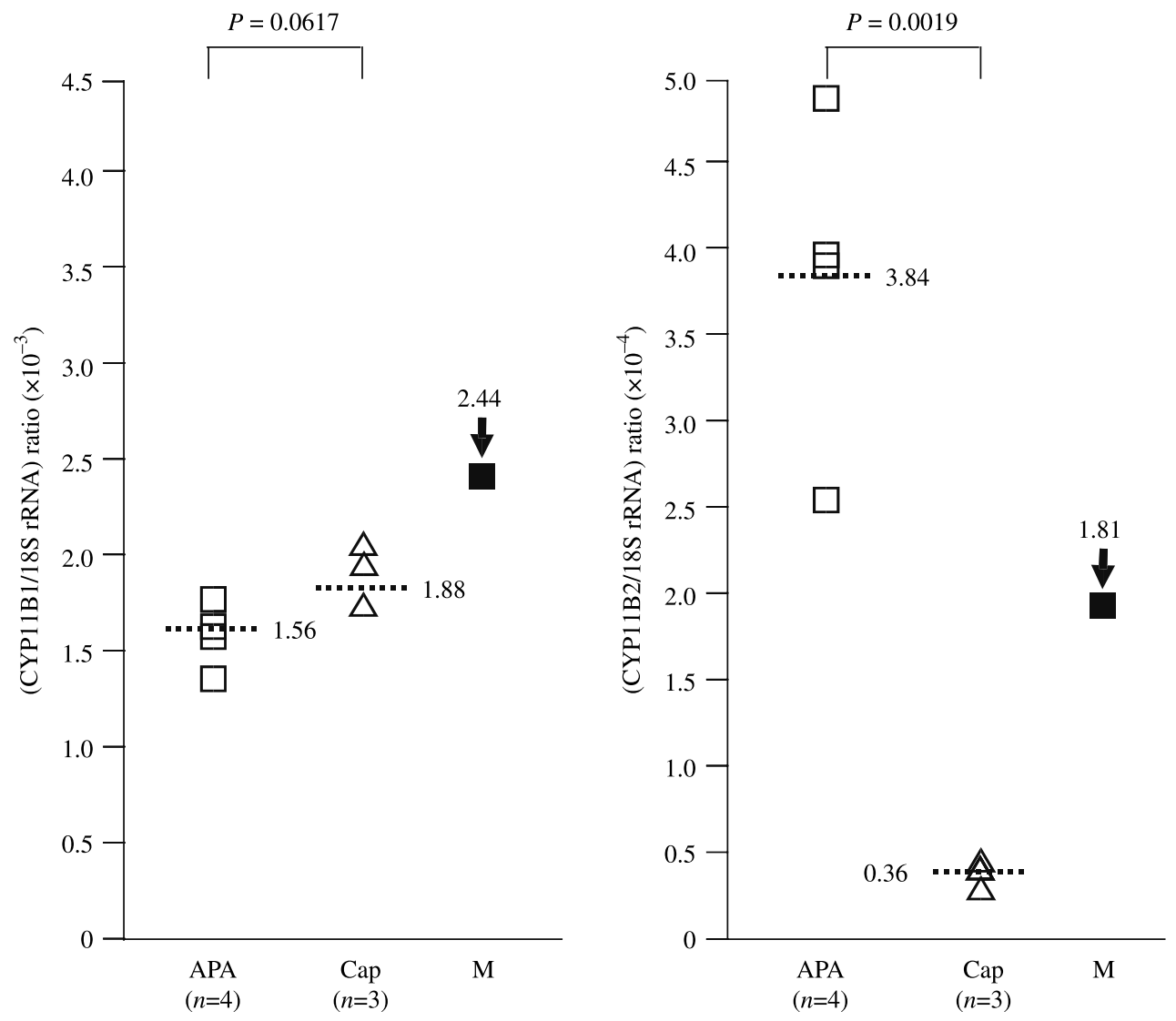

Figure 7 Ratios of CYP11B1/18S rRNA and CYP11B2/18S rRNA mRNAs. The results are shown as the ratio of CYP11B1 and CYP11B2 to $18 S$ rRNA. A dotted line shows the mean value in APA tissues and the capsular domain tissues without micronodule respectively. Student's $t$-test was used to compare APA tissues with the capsular domain tissues without subcapsular micronodules. Cap, the capsular domain tissues without subcapsular micronodules; $M$, the tissues containing subcapsular micronodule.

ZF-ZR (8). Vascular sclerosing is a frequent finding in PA and CS. Cortical nodules are also frequently found in the cortices attached to APA, CPA, and NFA (8). In this study, most of the nodules in ZF-ZR showed a decreased expression for steroidogenic enzymes. However, some were expressing all the necessary steroidogenic enzymes for cortisol production. We also demonstrated that adrenal cortical hyperplasia, with extension of cortical cells into the periadrenal fat, was composed of clear-type cells expressing HSD3B2 but not CYP17A1 and compacttype cells expressing CYP17A1 but not HSD3B2. Such differential expression of HSD3B2 and CYP17A1 has been reported only in ACTH-independent macronodular adrenocortical hyperplasia (32). Our results were consistent with those of previous reports showing the possibility that some nodules in the adrenal cortices of aging rats might be functional $(9,10)$. It is generally thought that cortical nodules occur as a result of compensatory local tissue hyperplasia following ischemic damage (33). As is known, ACTH is an important factor to stimulate the growth of the adrenal cortex through MC2R. Hence, we examined MC2R mRNA expression in the cortical nodules. MC2R mRNA was observed in the majority of APA cases, but absent in most of the CPA and NFA cases. Therefore, the nodules in the cortices of CPA or NFA were ACTH independent, although those in the cortices attached to APA were considered to be still under ACTH regulation. At present, these nodules are generally thought to be mere distortions of the architecture of the cortex rather than clonal neoplastic growths, such as adenomas and carcinomas (33). Certainly, the relapse rate after adrenalectomy is extremely low. One possible explanation is that the function of most nodules indicated in this study may be episodic or transient. Another possibility is that a long time may be required for the nodules to develop sufficiently to cause endocrine disruption. Finally, the participation of factors other than ACTH may be required for symptoms to be produced. However, the possibility that some nodules in the cortices attached to adenoma may develop into autonomous lesions causing CS at a future point cannot be completely excluded, because relapse even after total adrenalectomy has been reported $(3,16)$, although rarely.

Laparoscopic unilateral adrenalectomy is now the accepted method of surgery for unilateral benign adrenal tumor, but the choice between total and subtotal adrenalectomy remains controversial $(5,7$, 14, 17). Ishidoya et al. (17) experienced cases in which 
hypertension with endocrinological abnormality remained after subtotal adrenalectomy, and hence warned about the risk of subtotal adrenalectomy. It is thus necessary to keep in mind that lesions with the potential to later develop into functional adrenocortical nodules may be present in the remaining adrenal cortices, if subtotal adrenalectomy to remove only the adrenal mass is chosen.

\section{Acknowledgements}

The authors thank Mrs Amanda Nishida for critical comments and Miss Kanako Egashira for technical assistance. The authors did not receive any funding concerning this manuscript, and declare that there is no conflict of interest that would prejudice its impartiality.

\section{References}

1 Adachi J, Hirai Y, Terui K, Nakano T, Fukuda Y, Suda T \& Sasano H. A report of 7 cases of adrenal tumors secreting both cortisol and aldosterone. Internal Medicine 200342 714-718.

2 Shigematsu K, Irie J, Sakai H, Igawa T \& Kawai K. A case of adrenocortical adenoma with co-existent of primary aldosteronism and Cushing's syndrome. Shindan Byouri 200522 48-53.

3 Okura T, Miyoshi K, Watanabe S, Kurata M, Irita J, Manabe S, Fukuoka T, Higaki J \& Sasano H. Coexistence of three distinct adrenal tumors in the same adrenal gland in a patient with primary aldosteronism and preclinical Cushing's syndrome. Clinical and Experimental Nephrology 200610 127-130.

4 Satoh F, Murakami O, Takahashi K, Ueno J, Nishikawa T, Abe K, Mouri T \& Sasano H. Double adenomas with different pathological and hormonal features in the left adrenal gland of a patient with Cushing's syndrome. Clinical Endocrinology 199746 227-234.

5 Saito T, Ikoma A, Saito T, Tamemoto H, Suminaga Y, Yamada S, Kawakami M, Suzuki T, Sasano H \& Ishikawa S. Possibly simultaneous primary aldosteronism and preclinical Cushing's syndrome in a patient with double adenoma of right adrenal gland. Endocrine Journal 200754 287-293.

6 Aiba M, Kawakami M, Ito Y, Fujimoto Y, Suda T \& Demura H. Bilateral adrenocortical adenomas causing Cushing's syndrome. Archives of Pathology and Laboratory Medicine 1992116 146-150.

7 Nomura K, Saito H, Aiba M, Iihara M, Obara T \& Takano K. Cushing's syndrome due to bilateral adrenocortical adenomas with unique histological features. Endocrine Journal 2003 50 155-162.

8 Dobbie JW. Adrenocortical nodular hyperplasia: the ageing adrenal. Journal of Pathology 196999 1-18.

9 Kawai K, Sugihara H \& Tsuchiyama H. Histochemical observations on nodular portions in adrenal cortex of aging rats. Acta Histochemica et Cytochemica 19725 161-168.

10 Sugihara H, Kawai K \& Tsuchiyama H. Pathology of intracortical nodules in rat adrenal glands, especially on their fine-structure. Acta Histochemica et Cytochemica 197323 253-260.

11 Shigematsu K, Kawai K, Irie J, Sakai H, Nakashima O, Iguchi A, Shimamatsu J, Shimamatsu K, Kusaba Y \& Takahara O. Analysis of unilateral adrenal hyperplasia with primary aldosteronism from the aspect of messenger ribonucleic acid expression for steroidogenic enzymes: a comparative study with adrenal cortices adhering to aldosterone-producing adenoma. Endocrinology $2006147999-1006$.
12 Nakada T, Kubota Y, Sasagawa I, Yagisawa T, Watanabe M \& Ishigooka M. Therapeutic outcome of primary aldosteronism: adrenalectomy versus enucleation of aldosterone-producing adenoma. Journal of Urology 1995153 1775-1780.

13 Walz MK, Peitgen K, Diesing D, Petersenn S, Janssen OE, Philipp T, Metz KA, Mann K, Schmid KW \& Neumann HP. Partial versus total adrenalectomy by the posterior retroperitoneoscopic approach: early and long-term results of 325 consecutive procedures in primary adrenal neoplasias. World Journal of Surgery 200428 1323-1329.

14 Jeschke K, Janetschek G, Pesche R, Schellander L, Bartsch G \& Henning K. Laparoscopic partial adrenalectomy in patients with aldosterone-producing adenomas: indications, technique, and results. Urology $2003 \mathbf{6 1} 69-72$.

15 Calvo-Romero JM \& Ramos-Salado JL. Recurrence of adrenal aldosterone-producing adenoma. Postgraduate Medical Journal 200676 160-161.

16 Tung S-C, Wang P-W, Huang T-L, Lee W-C \& Chen W-J. Bilateral adrenocortical adenomas causing ACTH-independent Cushing's syndrome at different periods: a case report and discussion of corticosteroid replacement therapy following bilateral adrenalectomy. Journal of Endocrinological Investigation 200427 375-379.

17 Ishidoya S, Ito A, Sakai K, Satoh M, Chiba Y, Sato F \& Arai Y. Laparoscopic partial versus total adrenalectomy for aldosterone producing adenoma. Journal of Urology $2005 \mathbf{1 7 4} 40-48$.

18 Shigematsu K, Toriyama K, Kawai K \& Takahara O. Ectopic adrenal tissue in the thorax: a case report with in situ hybridization and immunohistochemical studies. Pathology, Research and Practice 2007203 543-548.

19 Mornet E, Dupont J, Vitek A \& White PC. Characterization of two genes encoding human steroid 11 $\beta$-hydroxylase (P-450 (11) $\beta$ ). Journal of Biological Chemistry $1989 \mathbf{2 6 4} 20961-20967$.

20 Liang F, Kapoun AM, Lam A, Damm DL, Quan D, O'Connell M \& Protter AA. B-type natriuretic peptide inhibited angiotensin II-stimulated cholesterol biosynthesis, cholesterol transfer, and steroidogenesis in primary human adrenocortical cells. Endocrinology $2007 \mathbf{1 4 8} 3722-3729$.

21 Ishimura $\mathrm{K} \&$ Fujita $\mathrm{H}$. Light and electron microscopic immunohistochemistry of the localization of adrenal steroidogenic enzymes. Microscopy Research and Technique 199736 445-453.

22 Midorikawa S, Sanada H, Hashimoto S, Suzuki T, Watanabe T \& Sasano H. Analysis of cortisol secretion in hormonally inactive adrenocortical incidentalomas: study of in vitro steroid secretion and immunohistochemical localization of steroidogenic enzymes. Endocrine Journal $2001 \mathbf{4 8} 167-174$.

23 Sasano H, Sato F, Shizawa S, Nagura H \& Coughtrie MW. Immunolocalization of dehydroepiandrosterone sulfotransferase in normal and pathologic human adrenal gland. Modern Pathology 19958 891-896.

24 Neville AM \& O'Hare MJ. Functional zonation of the adrenal cortex. In The Human Adrenal Cortex, (ch 10) pp 105-111. Eds AM Neville \& MJ O'Hare, Berlin, Heidelberg, New York: SpringerVerlag, 1982.

25 Rácz K, Pinet F, Marton T, Szende B, Gláz E \& Corvol P. Expression of steroidogenic enzyme messenger ribonucleic acids and corticosteroid production in aldosterone-producing and 'nonfunctioning' adrenal adenomas. Journal of Clinical Endocrinology and Metabolism 199377 677-682.

26 Fujii H, Kamide K, Miyake O, Abe T, Nagai M, Nakahama H, Horio T, Takiuchi S, Okuyama A, Yutani C \& Kawano Y. Primary aldosteronism combined with preclinical Cushing's syndrome in an elderly patient. Circulation Journal $2005691425-1427$.

27 Odagiri E, Naruse M, Terasaki K, Yamaguchi N, Jibiki K, Takagi S, Tanabe M \& Takano K. The diagnostic standard of preclinical Cushing's syndrome: evaluation of the dexamethasone suppression test using various cortisol kits. Endocrine Journal 2004 51 295-302.

28 Young WF Jr, Stanson AW, Grant CS, Thompson GB \& van Heerden JA. Primary aldosteronism: adrenal venous sampling. Surgery $1996 \mathbf{1 2 0} 913-920$. 
29 Kagawa CM, Cella JA \& Van Arman CG. Action of new steroids in blocking effects of aldosterone and deoxycorticosterone on salt. Science 1957126 1015-1016.

30 Cheng SC, Suzuki K, Sadee W \& Harding BW. Effects of spironolactone, canrenone and canrenoate-K on cytochrome P450, and 11beta- and 18-hydroxylation in bovine and human adrenal cortical mitochondria. Endocrinology 197699 1097-1106.

31 Enberg U, Volpe C, Hoog A, Wedell A, Farnebo L-O, Thoré M \& Hamberger B. Postoperative differentiation between unilateral adrenaladenoma and bilateral adrenal hyperplasia in primary aldosteronism by mRNA expression of the gene CYP11B2. European Journal of Endocrinology 2004151 73-85.
32 Sasano H, Suzuki T \& Nagura H. ACTH-independent macronodular adrenocortical hyperplasia: immunohistochemical and in situ hybridization studies of steroidogenic enzymes. Modern Pathology 19947 215-219.

33 Hornsby PJ. Aging of the human adrenal cortex. Science of Aging Knowledge Environment 200435 re6.

Received 8 February 2008

Accepted 21 February 2008 